Frontiers in Heat and Mass Transfer

\title{
HALL AND ION SLIP EFFECTS ON FREE CONVECTION HEAT AND MASS TRANSFER OF CHEMICALLY REACTING COUPLE STRESS FLUID IN A POROUS EXPANDING OR CONTRACTING WALLS WITH SORET AND DUFOUR EFFECTS
}

\author{
Odelu Ojjela*, N. Naresh Kumar \\ Department of Applied Mathematics \\ Defence Institute of Advanced Technology, Deemed University, Pune - 411025, India.
}

\begin{abstract}
This article deals the Hall and ion slip currents on free convection flow, heat and mass transfer of an electrically conducting couple stress fluid through porous channels with chemical reaction, Soret and Dufour effects. Assume that there is symmetric suction or injection along the expanding or contracting walls, which are maintained at different constant temperatures and concentrations. The governing partial differential equations are reduced to nonlinear dimensionless ordinary differential equations using the similarity transformations and solved numerically by the method of quasilinearization. The effects of various parameters on non-dimensional velocity components, temperature distribution and concentration are discussed in detail and shown in the form of graphs.
\end{abstract}

Keywords: MHD; Couple stress fluid; Free convection; Chemical reaction; Porous medium; Soret and Dufour.

\section{INTRODUCTION}

The MHD flow through porous channels has attracted the attention of several researchers due to their applications in science and engineering fields. Examples of these were found in the transport of biological flows between expanding or contracting vessels, the synchronous pulsation of porous diaphragms, MHD power generators, magnetic filtration and separation, jet printers, micro fluidic devices, polymeric liquids, boundary layer control, etc. The couple stress fluid theory developed by Stokes (1966) presents the generalization of the classical viscous fluid theory that sustains couple stresses and the body couples. The concept of couple stresses takes place due to the mechanical interactions in the fluid medium and in this theory the velocity field was defined in place of rotational field. Further Stokes (1968) discussed the effect of couple stresses through hydromagnetic channels in a fluid medium.

Many researchers studied the fluid flow in porous channels with expanding or contracting walls. Srinivasacharya et al. (2009) considered the problem on couple stress fluid flow and heat transfer in a porous channel with expanding and contracting walls and the solution was discussed using quasilinearization method. Alam et al. (2013) studied the couple stress fluid flow between expanding or contracting channel and an analytical approximate solution obtained for reduced governing equations. Analytical solutions were obtained for two dimensional incompressible symmetric and asymmetric flow of viscous fluid through porous channels between expanding or contracting walls discussed by Si et al. (2010 and 2011). Uchida and Akoi (1977) modeled the unsteady incompressible viscous fluid flows inside expanding or contracting pipe. When heat and mass transfer occur simultaneously in a moving fluid, it can be observed that an energy flux can be created not only by temperature gradients but also by concentration gradients. The energy flux caused by a concentration gradient is termed the Dufour effect. On the other hand, mass fluxes can also be generated by temperature gradients are known as the Soret effect. These effects are studied as second order phenomena and had applications in many areas such as petrology, geosciences, hydrology, etc. An incompressible flow, heat and mass transfer of a viscous fluid between expanding or contracting walls is considered by Srinivas et al. (2012) with weak permeability, Soret and Dufour effects. Srinivasacharya and Kaladhar (2011) examined the steady mixed convection heat and mass transfer of couple stress fluid past a vertical plate. An incompressible flow of couple stress liquids with Soret and Dufour effects studied by Malasetty et al. (2006).

Dursunkaya and Worek (1992) considered the diffusion-thermo and thermal-diffusion effects in transient and steady free convection flow over a vertical surface. The effects of thermal diffusion and diffusion thermo on the mixed convection heat and mass transfer of a non-Newtonian fluid in a porous medium with thermal radiation effect was investigated by Mahmoud and Megahed (2013). Nithyadevi and Yang (2009) examined numerically the Soret and Dufour effects on the free convection flow of water over a partially heated enclosure. Postelnicu (2004) considered the problem of MHD free convection flow, heat and mass transfer over a vertical surface with Soret and Dufour effects. The steady laminar incompressible mixed convection heat and mass transfer of viscous fluid embedded in the non Darcy porous medium over a stretching sheet with chemical reaction, Soret and Dufour effects analyzed by Dulal and Mondal (2011). Ganeshan et al. (2012) investigated the chemical reaction effect on natural convection flow over a vertical plate with Soret and Dufour effects. The steady free convection heat and mass transfer from the vertical cone with Soret and Dufour effects considered by Cheng (2010) and taking variable temperature and concentration.

In most of the electrically conducting fluid flow problems, the Hall and Ion-slip terms in Ohms law were neglected, then the influence of Hall current and Ion-slip are important under the presence of strong magnetic field. The effects of Soret and Dufour on the mixed convection heat and mass transfer of second grade fluid over a

*Corresponding author Email: odelu3@yahoo.co.in ; odelu@diat.ac.in 
stretching sheet is studied with Hall and ion slip currents by Hayat and Nawaz (2011). Elgazery (2009) examined the flow, heat and mass transfer of an electrically conducting viscous fluid with chemical reaction, Hall and ion slip by considering temperature dependent thermal diffusivity and viscosity. An unsteady magnetohydrodynamic natural convection heat and mass transfer from vertical surface with chemical reaction and radiation effects investigated by Chamkha et al. (2011). Patil and Kulkarni (2008) discussed the effect of chemical reaction on convective flow and heat transfer of polar fluid in a porous medium with internal heat generation. The application for quasilinearization technique to the flow of viscoelastic fluid in a porous annulus presented by Bhatnagar et al. (1994).

This paper investigates the influence of Hall and ion slip on unsteady two dimensional MHD natural convection heat and mass transfer of couple stress fluid in a porous medium between expanding or contracting walls with chemical reaction, Soret and Dufour effects. The reduced flow field equations are solved by using the quasilinearization method. The results are obtained in the form of graphs and discussed in detail for the velocity components, temperature distribution and concentration with respect to various fluid and geometric parameters.

\section{FORMULATION OF THE PROBLEM}

Consider an unsteady laminar incompressible free convection flow of couple stress fluid in a porous medium through two parallel horizontal walls. Assume that both upper and lower walls have equal permeability and expand or contract at a time dependent rate. Therefore their separation is a function of time $\mathrm{a}(\mathrm{t})$. The lower and upper walls are maintained at constant temperatures $\mathrm{T}_{1}, \mathrm{~T}_{2}$ and concentrations $\mathrm{C}_{1}, \mathrm{C}_{2}$ respectively. Let the fluid be injected or aspirated uniformly and orthogonally through the channel walls at an absolute velocity $\mathrm{V}_{1}$. The flow is subjected to a uniform magnetic field perpendicular to the flow direction with the Hall and ion-slip effects.

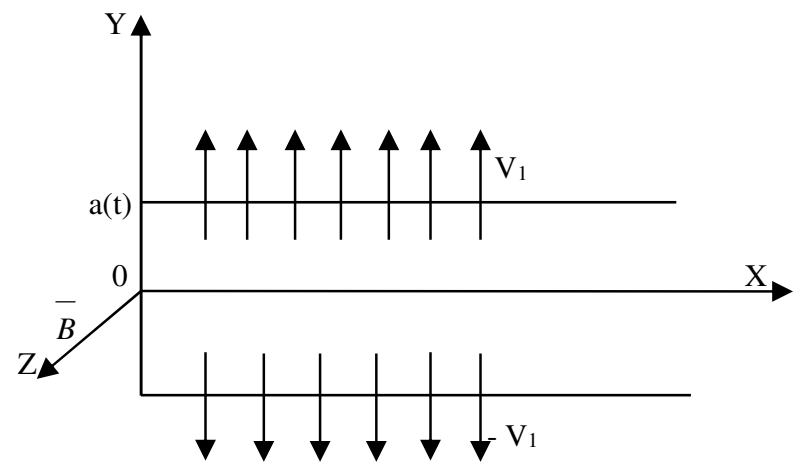

Fig. 1 Physical model of the problem

The equations governing the couple stress fluid flow, heat and mass transfer in the presence of magnetic field, Soret and Dufour effects and in the absence of body forces and body couples are given by

$$
\begin{aligned}
& \nabla \cdot \bar{Q}=0 \\
& \rho\left[\frac{\partial \bar{Q}}{\partial t}+(\bar{Q} \cdot \nabla) \bar{Q}\right]=-\operatorname{grad} p-\mu \operatorname{curl}(\operatorname{curl}(\bar{Q})) \\
& -\eta \operatorname{curl}(\operatorname{curl}(\operatorname{curl}(\operatorname{curl}(\bar{Q}))))-\frac{\mu}{k_{1}} \bar{Q}+\bar{J} \times \bar{B}+\overline{F_{B}}
\end{aligned}
$$

$\rho c\left[\frac{\partial T}{\partial t}+(\bar{Q} \cdot \nabla) T\right]=k \nabla^{2} T+2 \mu D: D+4 \eta\left[(\nabla \bar{\omega}):(\nabla \bar{\omega})^{T}\right]+$

$4 \eta^{\prime}[(\nabla \bar{\omega}):(\nabla \bar{\omega})]+\frac{\mu}{k_{1}}(\bar{Q})^{2}+\frac{(\bar{J})^{2}}{\sigma}+\frac{\rho D_{1} k_{T}}{c_{s}} \nabla^{2} C$

$\left[\frac{\partial C}{\partial t}+(\bar{Q} \cdot \nabla) C\right]=D_{1} \nabla^{2} C-k_{2}\left(C-C_{1}\right)+\frac{D_{1} k_{T}}{T_{m}} \nabla^{2} T$

where $\overline{F_{B}}$ is the buoyancy force and it is defined as $\left(\rho g \beta_{T}\left(T-T_{1}\right)+\rho \beta_{C}\left(C-C_{1}\right) \hat{)}, \bar{Q} \quad\right.$ is velocity, $\mathrm{p}$ is pressure, $\mathrm{k}_{1}$ is permeability parameter, $\sigma$ is electrical conductivity, $\rho$ is density, $\bar{B}$ is magnetic field, $\bar{E}$ is electric field, $\mathrm{T}$ is the temperature distribution, $\bar{\omega}$ is the rotation vector, $\mathrm{k}$ is the thermal conductivity, $\mathrm{c}$ is the specific heat at constant temperature, $k_{T}$ is the thermal diffusion ratio, $T_{m}$ is the mean temperature, $c_{s}$ is the concentration susceptibility, $D_{1}$ is the coefficient of mass diffusivity, $\eta$ and $\eta^{\prime}$ are the couple stress fluid parameters, $\mathrm{C}$ is the concentration, $\mathrm{k}_{2}$ is the chemical reaction rate and $\bar{J}$ is current density.

The force stress tensor $\tau$ and the couple stress tensor M that arises in the theory of couple stress fluids are given by

$$
\begin{aligned}
\tau= & (-p+\lambda \operatorname{div} \bar{Q}) I+\mu\left[\operatorname{grad} \bar{Q}+(\operatorname{grad} \bar{Q})^{T}\right]+ \\
& 1 / 2 I[\operatorname{div} M+\rho C]
\end{aligned}
$$

and

$M=m I+2 \eta \operatorname{grad}(\operatorname{curl} \bar{Q})+2 \eta^{\prime}(\operatorname{grad}(\operatorname{curl} \bar{Q}))^{T}$

where $\mathrm{m}$ is $1 / 3^{\text {rd }}$ Trace of $M$ and $\rho C$ is the body couple tensor. The quantity $\lambda$ is the material constant and $\eta$ is the constant associated with couple stresses. The dimensions of the material constant $\lambda$ is that of viscosity where as the dimensions of $\eta$ and $\eta$ are those of momentum. These material constants are considered by the inequalities,

$\mu \geq 0, \quad 3 \lambda+2 \mu \geq 0, \eta \geq 0, \quad \eta \leq \eta$

Neglecting the displacement currents, the Maxwell equations and the generalized Ohm's law are

$$
\begin{aligned}
& \nabla \cdot \bar{B}=0, \quad \nabla \times \bar{B}=\mu^{\prime} \bar{J}, \quad \nabla \times \bar{E}=\frac{\partial \bar{B}}{\partial t}, \\
& \bar{J}=\frac{\hat{\sigma B_{0}}}{\alpha e^{2}+\beta e^{2}}((\alpha e v-\beta e u) \hat{i}-(\alpha e u+\beta e v) \hat{j})
\end{aligned}
$$

Where $\bar{B}=B_{0} \hat{k}+\bar{b}, \bar{b}$ is the induced magnetic field $\beta_{e}$ is the Hall parameter $\beta_{i}$ is the ion slip parameter and $\mu^{\prime}$ is magnetic permeability.

Let the induced magnetic field be negligible compared to the applied magnetic field so that magnetic Reynolds number is small, the electric field is zero and magnetic permeability is constant throughout the flow field.

The velocity is $\bar{Q}=u \hat{i}+v \hat{j}$,

Following Si et al. (2010 and 2011), we take the velocity components as, 
$u(x, \xi, t)=-\frac{v x}{a^{2}} F^{\prime}(\xi, t), v(x, \xi, t)=\frac{v}{a} F(\xi, t)$,

Following Srinivas et al. (2012), we take the temperature distribution as

$T(x, \xi, t)=T_{1}+\frac{\mu V_{1}}{\rho a c}\left(\varphi_{1}(\xi)+\frac{x^{2}}{a^{2}} \varphi_{2}(\xi)\right)$ and

$C(x, \xi, t)=C_{1}+\frac{n_{A}}{a v}\left(g_{1}(\xi)+\frac{x^{2}}{a^{2}} g_{2}(\xi)\right)$

Where $\xi=\frac{y}{a(t)}$ and $F(\xi, t), \varphi_{1}(\xi), \varphi_{2}(\xi), g_{1}(\xi), g_{2}(\xi)$ are to be determined.

The boundary conditions on the velocity, temperature and concentration are

$u(x, \xi, t)=0, v(x, \xi, t)=0, \nabla \times \bar{Q}=0, T(x, \xi, t)=T_{1,}$,

$C(x, \xi, t)=C_{1}$ at $\xi=0$

$u(x, \xi, t)=0, v(x, \xi, t)=V_{l}, \quad \nabla \times \bar{Q}=0, T(x, \xi, t)=T_{2}$,

$C(x, \xi, t)=C_{2}$ at $\xi=1$

Substituting (9) in (2), (3), and (4) then

$$
\begin{gathered}
f^{V \prime}=\frac{1}{\alpha^{2}}\left(\xi \beta f^{\prime \prime \prime}+3 \beta f^{\prime \prime}+\operatorname{Re}\left(f^{\prime} f^{\prime \prime}-f f^{\prime \prime \prime}\right)+f^{\prime V}-D^{-1} f^{\prime \prime}\right. \\
-\frac{H a^{2} \alpha e}{\alpha e^{2}+\beta e^{2}} f^{\prime \prime}-\frac{E G r}{\operatorname{Re} \zeta}\left(\varphi_{1}^{\prime}+\zeta^{2} \varphi_{2}^{\prime}\right)-\frac{S h G m}{\operatorname{Re} \zeta}\left(g_{1}^{\prime}+\zeta^{2} g_{2}^{\prime}\right)
\end{gathered}
$$

$\varphi_{1}^{\prime \prime}=-2 \varphi_{2}-\operatorname{Re} \operatorname{Pr}\left(4 f^{\prime^{2}}+\alpha^{2} f^{\prime /^{2}}+D^{-1} f^{2}+\frac{H a^{2}}{\alpha e^{2}+\beta e^{2}} f^{2}+\right.$

$$
\left.\frac{\beta \xi \varphi_{1}^{\prime}}{\operatorname{Re}}+\frac{\beta \varphi_{1}}{\operatorname{Re}}-f \varphi_{1}^{\prime}\right)-2 \operatorname{Du} g_{2}-\operatorname{Du} g_{1}^{\prime \prime}
$$

$\varphi_{2}^{\prime \prime}=-\operatorname{Re} \operatorname{Pr}\left(f^{/ /^{2}}+\alpha^{2} f^{/ / /^{2}}+D^{-1} f^{\prime^{2}}+\frac{H a^{2}}{\alpha e^{2}+\beta e^{2}} f^{\prime^{2}}+\right.$

$$
\left.\frac{\xi \beta g_{2}^{\prime}}{\operatorname{Re}}+\frac{3 \beta g_{2}}{\operatorname{Re}}+2 f^{\prime} \phi_{2}-f \phi_{2}^{\prime}\right)-\operatorname{Du} g_{2}^{\prime \prime}
$$

$$
\begin{aligned}
g_{1}^{\prime \prime}= & -2 g_{2}+\operatorname{Kr} g_{1}+S c\left(-\beta g_{1}-\xi \beta g_{1}^{\prime}+\operatorname{Re} f g_{1}^{\prime}\right) \\
& \quad-\operatorname{ScS} r\left(\varphi_{1}^{\prime \prime}+2 \varphi_{2}\right) \\
g_{2}^{\prime \prime}= & \operatorname{Kr} g_{1}+\operatorname{Sc}\left(-3 \beta g_{2}-\xi \beta g_{2}^{\prime}+\operatorname{Re}\left(f g_{2}^{\prime}-2 f^{\prime} g_{2}\right)\right) \\
& -\operatorname{ScS} \varphi_{2}^{\prime \prime}
\end{aligned}
$$

Where prime denotes the differentiation with respect to $\xi$, $f(\xi)=\frac{F(\xi, t)}{\operatorname{Re}}, \alpha e=1+\beta e \beta i$.

The dimensionless form of temperature and concentration from (9) can be written as

$$
\begin{aligned}
& T^{*}=\frac{T-T_{1}}{T_{2}-T_{1}}=E c\left(\varphi_{1}+\zeta^{2} \varphi_{2}\right) \\
& C=\frac{C-C_{1}}{C_{2}-C_{1}}=\operatorname{Sh}\left(g_{1}+\zeta^{2} g_{2}\right)
\end{aligned}
$$

Where $E c=\frac{\mu V_{1}}{\rho a c\left(T_{2}-T_{1}\right)}$ is the Eckert number,

$$
S h=\frac{n_{A}}{a v\left(C_{2}-C_{1}\right)} \text { is the Sherwood number and } \zeta=\frac{x}{a} \text { is the }
$$

dimensionless axial variable.

The boundary conditions (10) in terms of $f, \varphi_{1}, \varphi_{2}, g_{1}$ and $g_{2}$ are

$$
\begin{array}{cl}
f(0)=0, & f(1)=1, \\
f^{\prime}(0)=0, & f^{\prime}(1)=0, \\
f^{\prime \prime}(0)=0, & f^{\prime \prime}(1)=0, \\
\varphi_{1}(0)=0, & \varphi_{1}(1)=1 / E c \\
\varphi_{2}(0)=0, & \varphi_{2}(1)=0, \\
g_{1}(0)=0, & g_{1}(1)=1 / S h \\
g_{2}(0)=0, & g_{2}(1)=0
\end{array}
$$

\section{SOLUTION OF THE PROBLEM}

The nonlinear equations (11), (12), (13), (14) and (15) are converted into the following system of first order differential equations by the substitution

$\left(f, f^{\prime}, f^{\prime \prime}, f^{\prime \prime \prime}, f^{\prime V}, f^{V}, \varphi_{1}, \varphi_{1}^{\prime}, \varphi_{2}, \varphi_{2}^{\prime}, g_{1}, g_{1}^{\prime}, g_{2}, g_{2}^{\prime}\right)=$ $\left(x_{1}, x_{2}, x_{3}, x_{4}, x_{5}, x_{6}, x_{7}, x_{8}, x_{9}, x_{10}, x_{11}, x_{12}, x_{13}, x_{14}\right)$

$\frac{d x_{1}}{d \xi}=x_{2}, \frac{d x_{2}}{d \xi}=x_{3}, \frac{d x_{3}}{d \xi}=x_{4}, \frac{d x_{4}}{d \xi}=x_{5}, \frac{d x_{5}}{d \xi}=x_{6}$,

$\frac{d x_{6}}{d \xi}=\frac{1}{\alpha^{2}}\left(\xi \beta x_{4}+3 \beta x_{3}+\operatorname{Re}\left(x_{2} x_{3}-x_{1} x_{4}\right)+x_{5}-D^{-1} x_{3}\right.$

$\left.-\frac{H a^{2} \alpha e}{\alpha e^{2}+\beta e^{2}} x_{3}-\frac{E G r}{\operatorname{Re} \zeta}\left(x_{8}+\zeta^{2} x_{12}\right)-\frac{S h G m}{\operatorname{Re} \zeta}\left(x_{12}+\zeta^{2} x_{14}\right)\right)$,

$\frac{d x_{7}}{d \xi}=x_{8}$ 


$$
\begin{aligned}
& \frac{d x_{8}}{d \xi}=-2 x_{9}-\frac{\operatorname{Re} \operatorname{Pr}}{1-D u S c S r}\left(4 x_{2}^{2}+\alpha^{2} x_{3}^{2}+D^{-1} x_{1}^{2}+\right. \\
& \left.\frac{H a^{2}}{\alpha e^{2}+\beta e^{2}} x_{1}^{2}+\frac{\beta \xi x_{8}}{\operatorname{Re}}+\frac{\beta x_{7}}{\operatorname{Re}}-x_{1} x_{8}\right)-\frac{K r D u}{1-D u S c S r} x_{11}+ \\
& \frac{D u S c}{1-D u S c S r}\left(-\beta x_{11}-\xi \beta x_{12}+\operatorname{Re} x_{1} x_{12}\right), \\
& \frac{d x_{9}}{d \xi}=x_{10}, \\
& \frac{d x_{10}}{d \xi}=-\frac{\operatorname{Re} \operatorname{Pr}}{1-D u S c S r}\left(x_{3}^{2}+\alpha^{2} x_{4}^{2}+D^{-1} x_{2}^{2}+\frac{H a^{2}}{\alpha e^{2}+\beta e^{2}} x_{2}^{2}\right. \\
& \left.\frac{\xi \beta x_{10}}{\operatorname{Kr} D u}+\frac{3 \beta x_{9}}{\operatorname{Re}}+2 x_{2} x_{9}-x_{1} x_{10}\right)- \\
& \frac{\operatorname{Re}}{1-D u S c S r}+\frac{D u S c}{1-D u S c S r}\left(-3 \beta x_{13}-\xi \beta x_{14}+\operatorname{Re}\left(x_{1} x_{14}-2 x_{2} x_{13}\right)\right)
\end{aligned}
$$$$
\frac{d x_{11}}{d \xi}=x_{12}
$$$$
\frac{d x_{12}}{d \xi}=-2 x_{13}+\frac{S c S r \operatorname{Re} \operatorname{Pr}}{1-D u S c S r}\left(4 x_{2}^{2}+\alpha^{2} x_{3}^{2}+D^{-1} x_{1}^{2}+\right.
$$$$
\left.\frac{H a^{2}}{\alpha e^{2}+\beta e^{2}} x_{1}^{2}+\frac{\beta \xi x_{8}}{\operatorname{Re}}+\frac{\beta x_{7}}{\operatorname{Re}}-x_{1} x_{8}\right)+\frac{K r}{1-D u S c S r} x_{11}+
$$$$
\frac{S c}{1-D u S c S r}\left(-\beta x_{11}-\xi \beta x_{12}+\operatorname{Re} x_{1} x_{12}\right), \quad \frac{d x_{13}}{d \xi}=x_{14},
$$$$
\frac{d x_{14}}{d \xi}=\frac{S c S r \operatorname{Re} \operatorname{Pr}}{1-D u S c S r}\left(x_{3}^{2}+\alpha^{2} x_{4}^{2}+D^{-1} x_{2}^{2}+\frac{H a^{2}}{\alpha e^{2}+\beta e^{2}} x_{2}^{2}+\frac{\xi \beta x_{10}}{\operatorname{Re}}\right.
$$$$
\begin{aligned}
& \left.+\frac{3 \beta x_{9}}{\operatorname{Re}}+2 x_{2} x_{9}-x_{1} x_{10}\right)+\frac{K r}{1-D u S c S r} x_{13}+ \\
& \frac{S c}{1-D u S c S r}\left(-3 \beta x_{13}-\xi \beta x_{14}+\operatorname{Re}\left(x_{1} x_{14}-2 x_{2} x_{13}\right)\right)
\end{aligned}
$$

The boundary conditions in terms

of $x_{1}, x_{2}, x_{3}, x_{4}, x_{5}, x_{6}, x_{7}, x_{8}, x_{9}, x_{10}, x_{11}, x_{12}, x_{13}, x_{14}$ are

$x_{1}(0)=0, x_{2}(0)=0, x_{3}(0)=0, x_{7}(0)=0, x_{9}(0)=0$,

$x_{11}(0)=0, x_{13}(0)=0$,

$$
\begin{aligned}
& x_{1}(1)=1, x_{2}(1)=0, x_{3}(1)=0, x_{7}(1)=1 / E c, x_{9}(1)=0, \\
& x_{11}(1)=1 / S h, x_{13}(1)=0
\end{aligned}
$$

The system of equations (18) is solved numerically subject to the boundary conditions (19) using quasilinearization method given by Bellman and Kalaba (1965).

\section{SKIN FRICTION}

Using equation (5), the shear stress $\tau$ is

$$
\tau=\mu \frac{\partial u}{\partial y}
$$

Then the coefficient of skin friction on the lower plate is given by

$$
S_{f}=\left(\frac{2 \tau}{\rho V_{1}^{2}}\right)_{\xi=0}=\frac{2}{\operatorname{Re}} \frac{x}{a} f^{\prime \prime}(0)
$$

\section{RESULTS AND DISCUSSION}

The reduced flow field equations (11) to (15) are fully coupled nonlinear ordinary differential equations that is why we cannot solve analytically. Therefore, solving the above nonlinear equations using quasilinearization method, we obtained the solutions of non dimensional velocity components, temperature distribution and concentration for various fluid and geometric parameters such as Soret number Sr, Dufour number Du, Hall parameter $\beta$ e, ion slip parameter $\beta i$, chemical reaction parameter $\mathrm{Kr}$, thermal Grashof number $\mathrm{Gr}$ and solutal Grashof number Gm and presented in the form of graphs.

The effects of $\mathrm{Gr}$ and $\mathrm{Gm}$ are shown in the Figs. 2 and 3. The Gr and $\mathrm{Gm}$ have the relative effect of the thermal and solutal buoyancy force to the viscous hydrodynamic force at the boundary layer. Hence their effects on velocity components and temperature are one and same. i.e., When $\mathrm{Gr}$ and $\mathrm{Gm}$ are increasing the radial velocity is also increasing, whereas the temperature distribution is decreasing and the axial velocity increases towards the center of the walls then decrease.

Figures 4 and 5 show the effects of $\beta \mathrm{e}$ and $\beta \mathrm{i}$ on velocity components, temperature distribution and concentration and from these it is analyzed that the concentration is increasing, whereas the radial velocity and temperature distribution are decreasing with the increasing of $\beta \mathrm{e}$ and $\beta \mathrm{i}$. However, the axial velocity decreases towards the center of the plane then increases. It is because of the increase in the Hall and ion slip parameters reducing the effect of Lorentz force.

Figures 6 and 7 depict the variations of $\mathrm{Sr}$ and $\mathrm{Du}$ on temperature distribution and concentration. When $\mathrm{Sr}$ and $\mathrm{Du}$ increase the temperature distribution also increases, whereas the concentration decreases. This is because of an increase in $\mathrm{Sr}$ and $\mathrm{Du}$ increase the difference between the temperature of the fluid and the surface temperature and also increase in the difference between the surface concentration and the concentration of the fluid.

The effect of $\mathrm{Kr}$ on temperature distribution and concentration are presented in the Fig. 8. It is observed that when $\mathrm{Kr}$ is increasing the temperature distribution is also increasing, whereas concentration is decreasing towards the upper wall. It is clear that the increase in the $\mathrm{Kr}$ produces a decrease in the species concentration. This causes the concentration buoyancy effects to decrease as $\mathrm{Kr}$ increases. In addition the concentration boundary layer thickness decreases as $\mathrm{Kr}$ increases. 


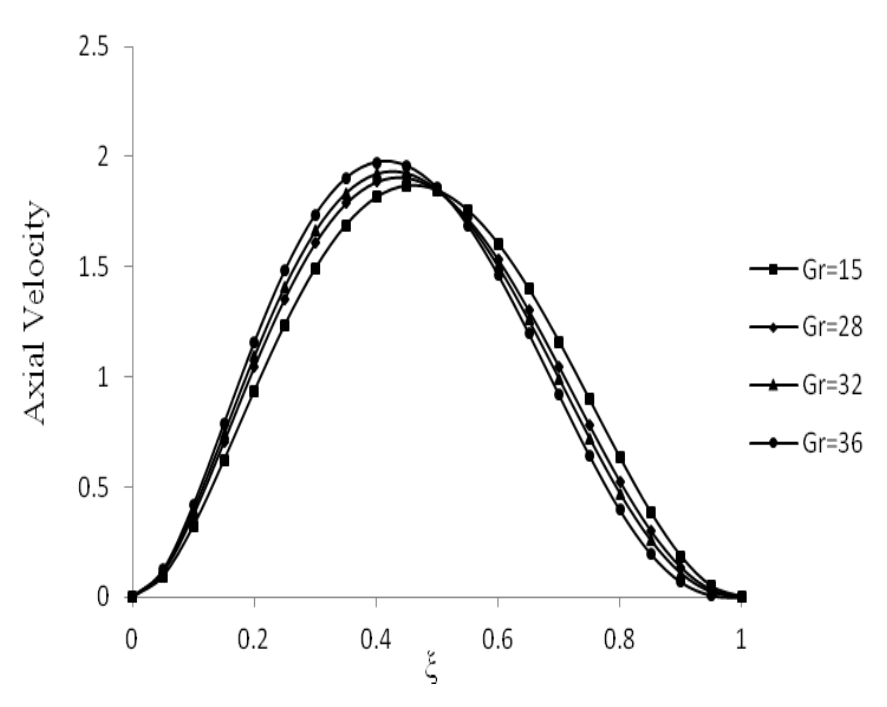

(a)

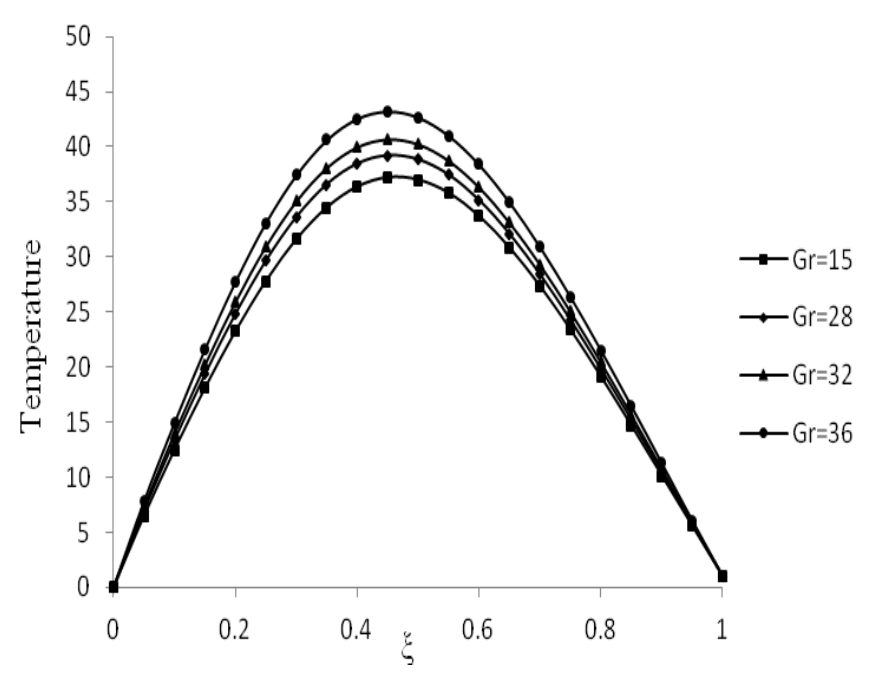

(c)

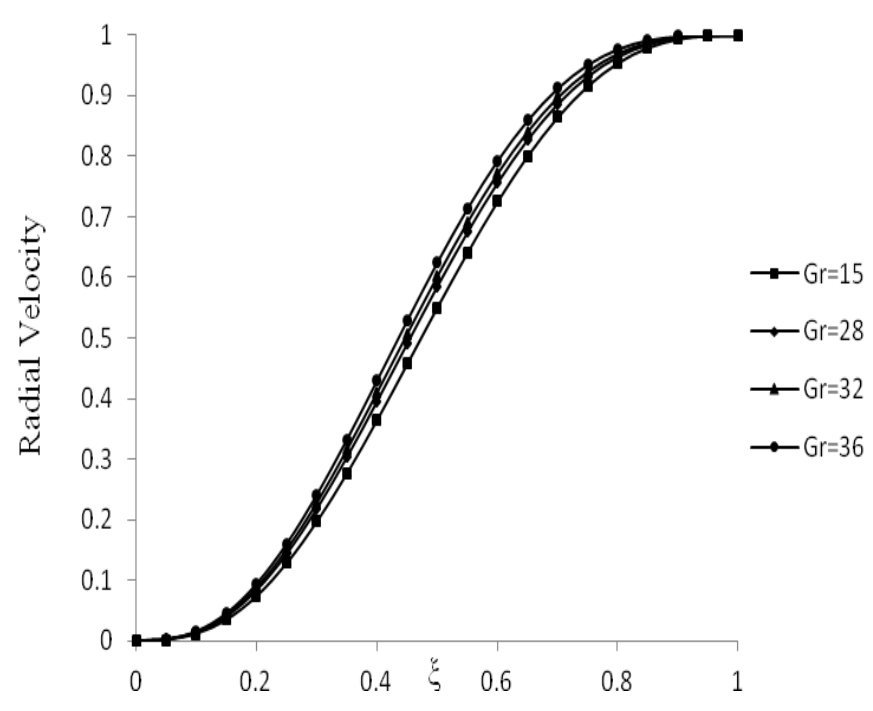

(b)

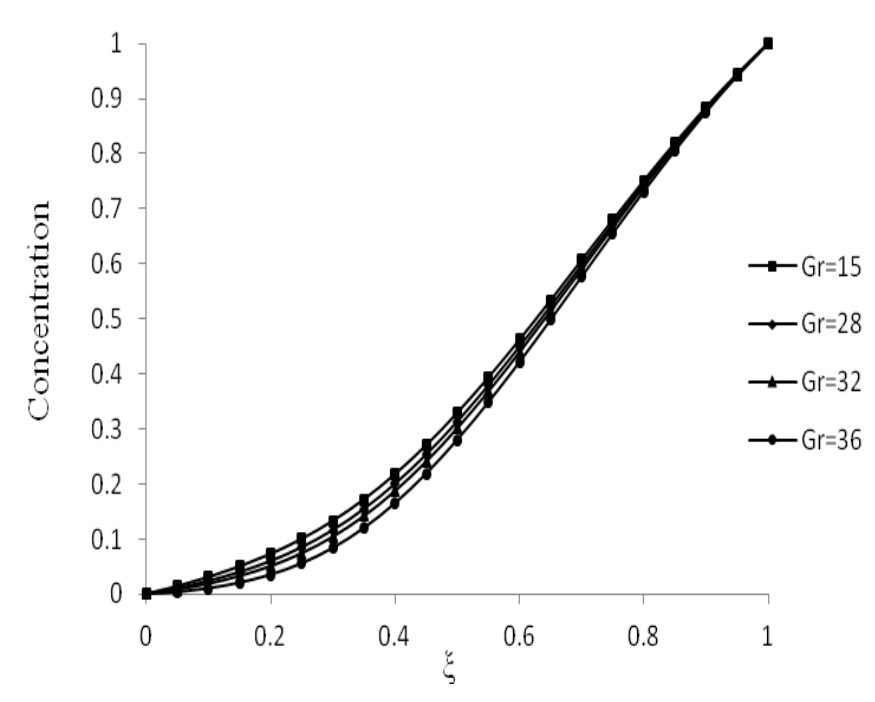

(d)

Fig. 2 Effect of Gr on (a) Axial Velocity, (b) Radial Velocity, (c) Temperature and (d) Concentration for $\mathrm{Kr}=2, \beta \mathrm{i}=0.2, \mathrm{Gm}=20, \mathrm{Sr}=0.02, \mathrm{Du}=0.2, \beta \mathrm{e}=0.2, \mathrm{Sc}=0.22, \mathrm{Pr}=0.2, \mathrm{Re}=2, \mathrm{D}^{-1}=2, \beta=10, \alpha=0.5, \mathrm{Ha}=10$. 


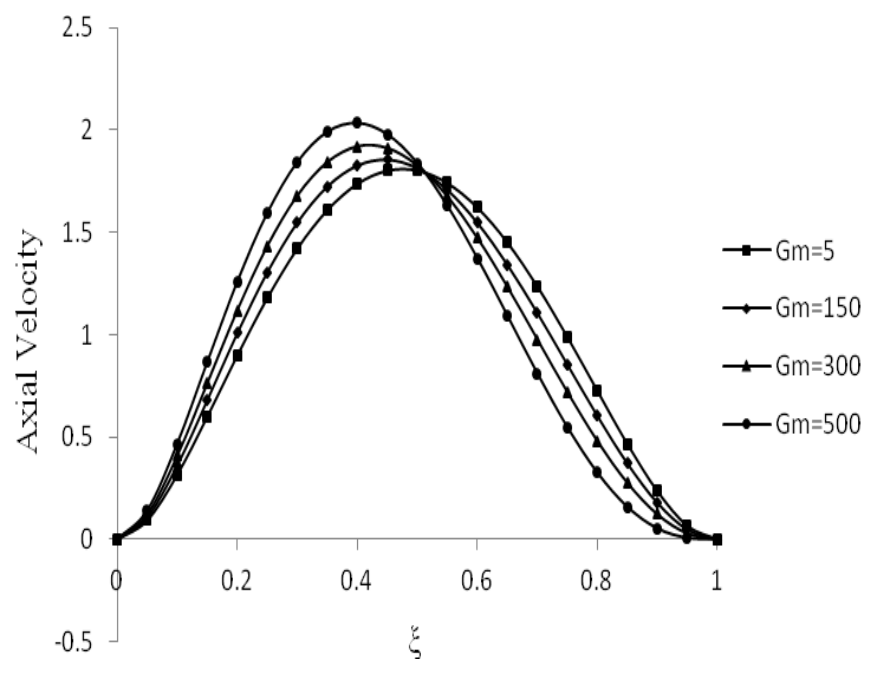

(a)

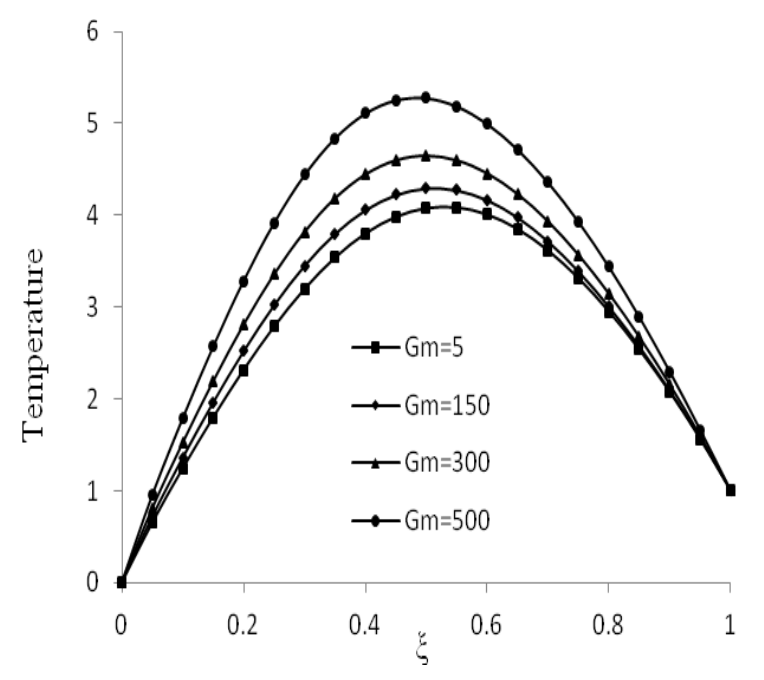

(c)

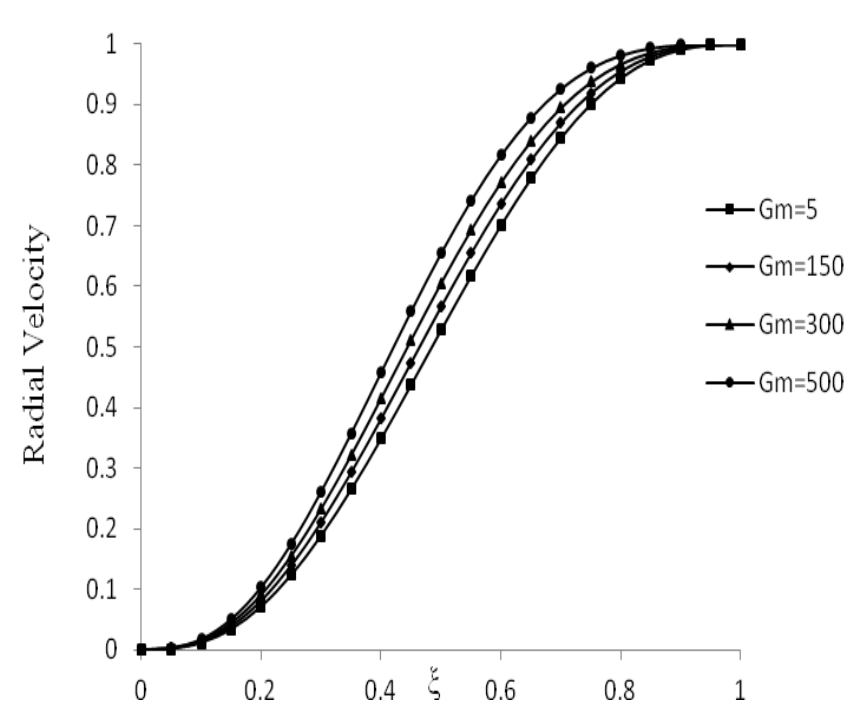

(b)

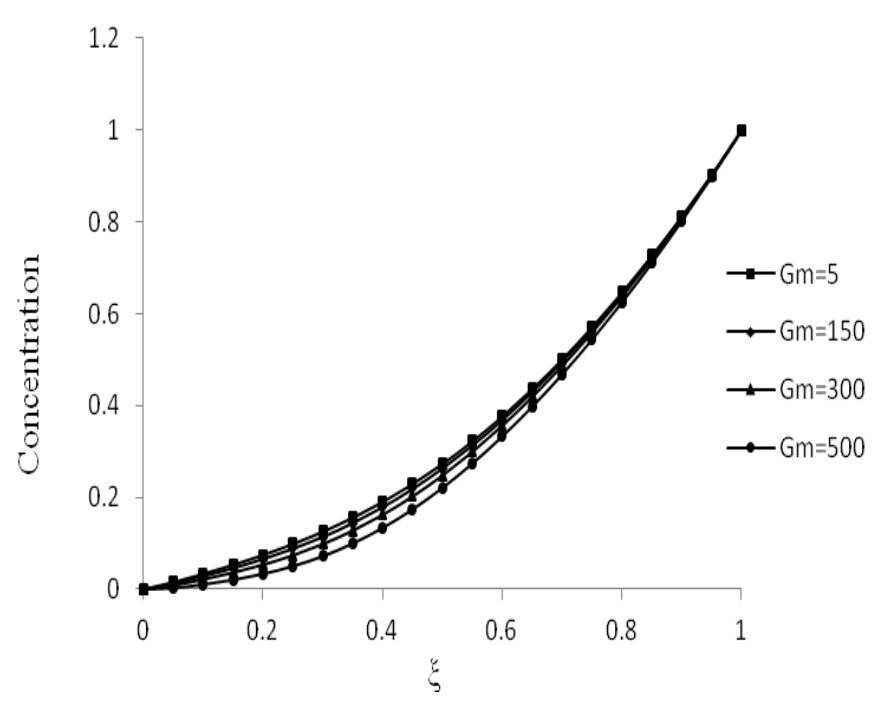

(d)

Fig. 3 Effect of Gm on (a) Axial Velocity, (b) Radial Velocity, (c) Temperature and (d) Concentration for $\mathrm{Kr}=2, \beta \mathrm{i}=0.2, \mathrm{Gr}=25, \mathrm{Sr}=0.2, \mathrm{Du}=0.2, \beta \mathrm{e}=0.2, \mathrm{Sc}=0.2, \mathrm{Pr}=0.2, \mathrm{Re}=2, \mathrm{D}^{-1}=10, \beta=2, \alpha=0.2, \mathrm{Ha}=2$. 


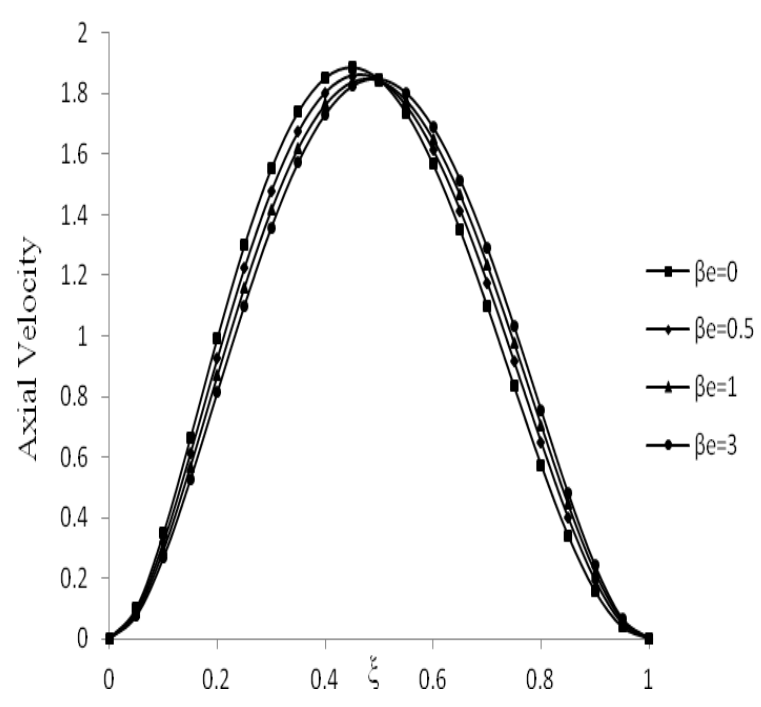

(a)

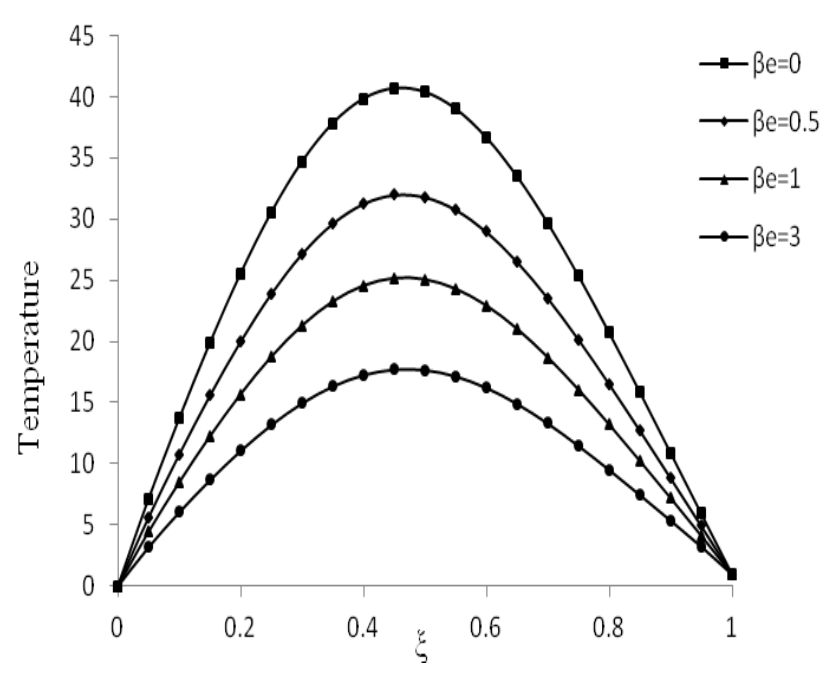

(c)

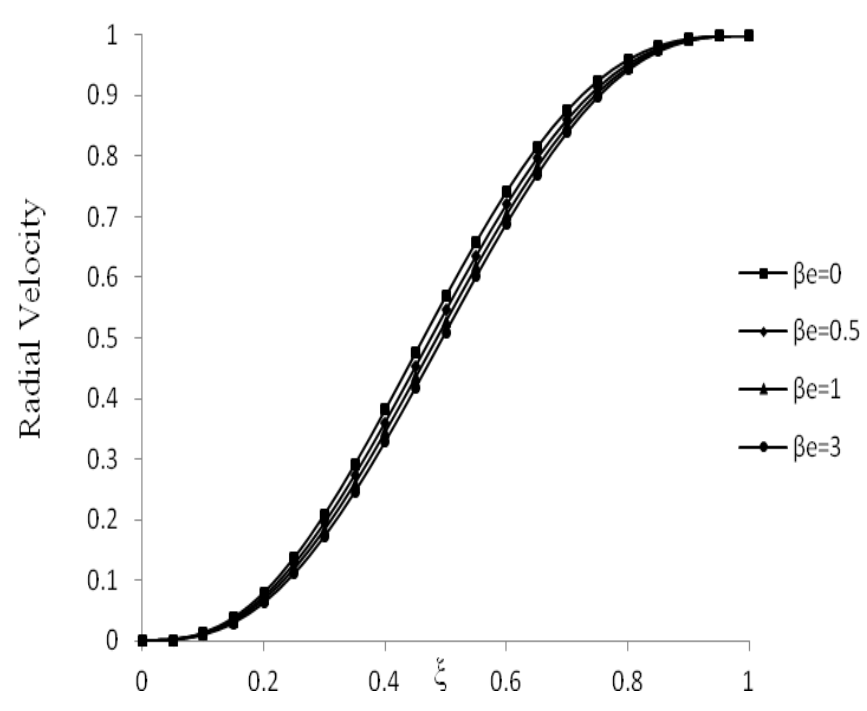

(b)

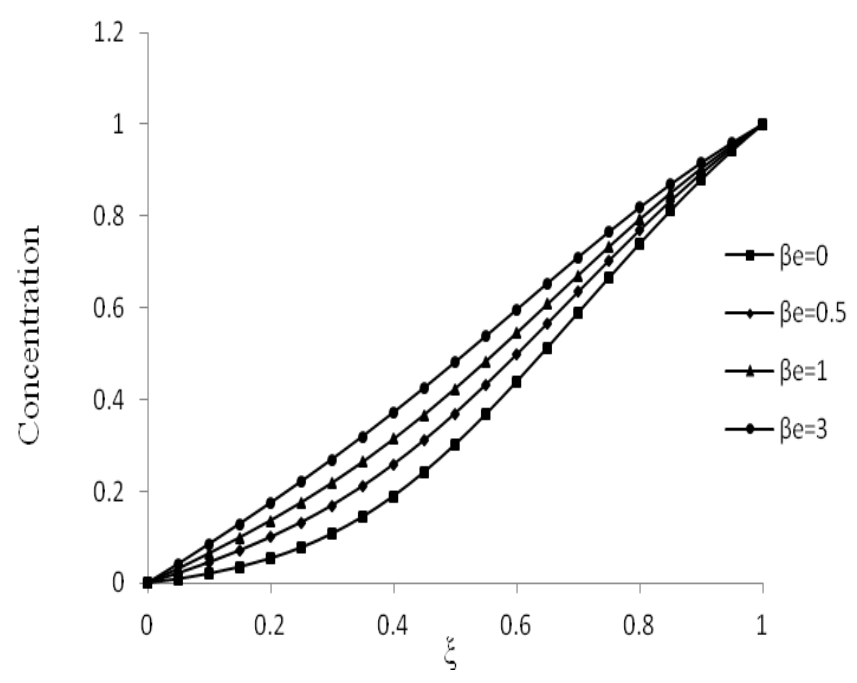

(d)

Fig. 4 Effect of $\beta e$ on (a) Axial Velocity, (b) Radial Velocity, (c) Temperature and (d) Concentration for $\mathrm{Kr}=2, \beta \mathrm{i}=0.2, \mathrm{Gr}=20, \mathrm{Sr}=0.02, \mathrm{Du}=0.2, \mathrm{Gm}=20, \mathrm{Sc}=0.22, \mathrm{Pr}=0.2, \mathrm{Re}=2, \mathrm{D}^{-1}=2, \beta=10, \alpha=0.5, \mathrm{Ha}=10$. 


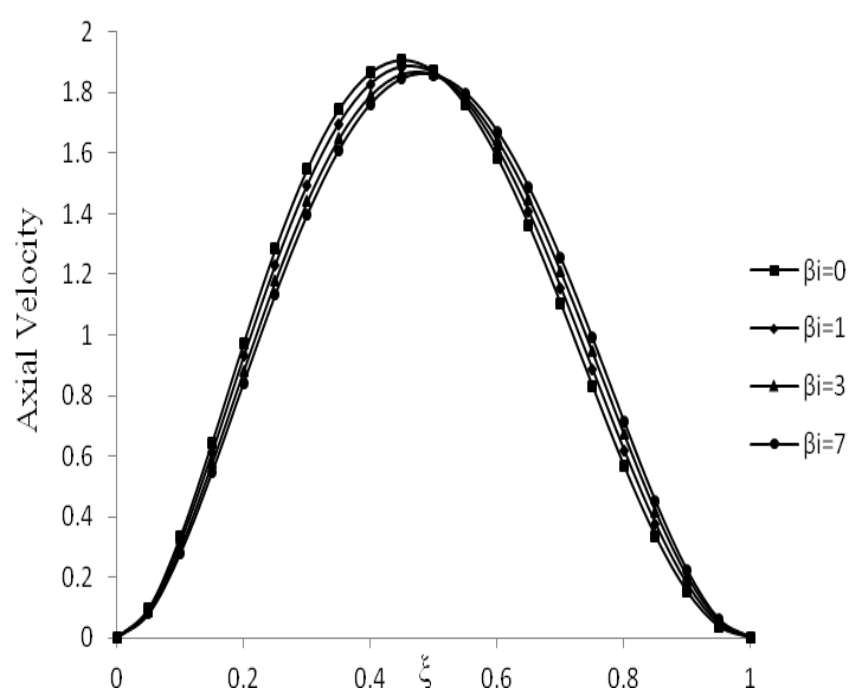

(a)

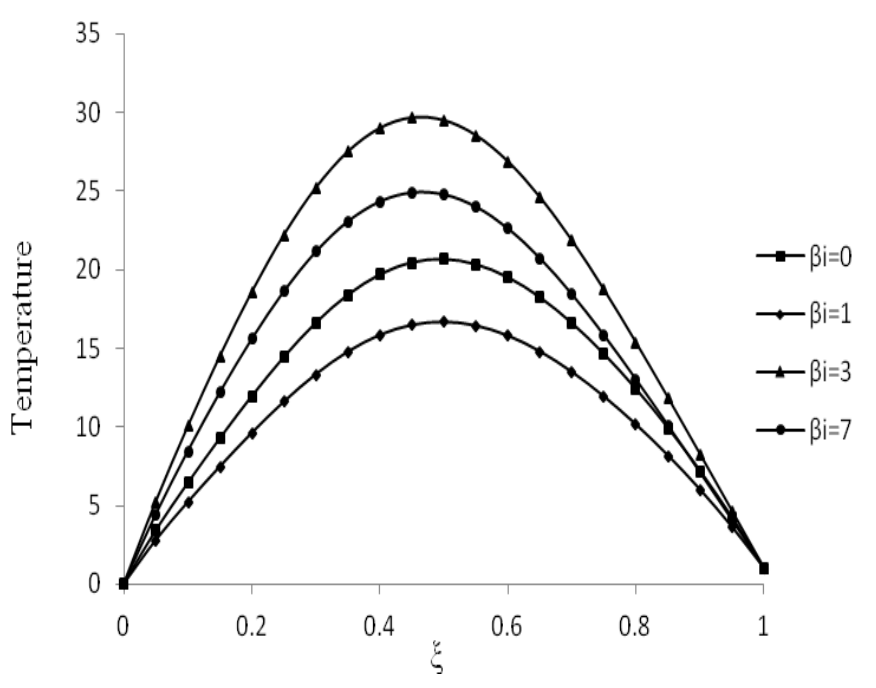

(c)

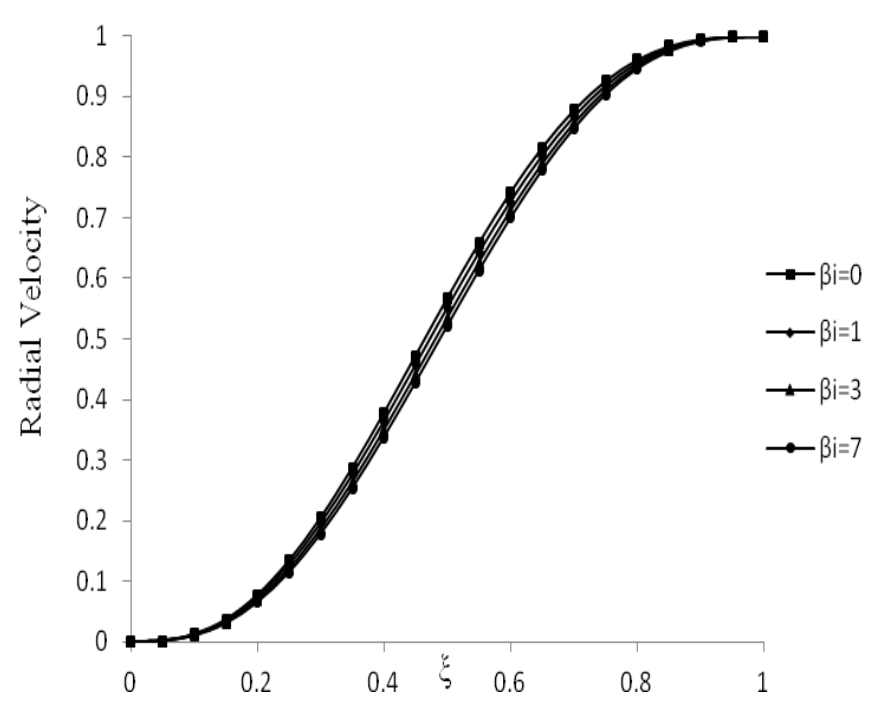

(b)

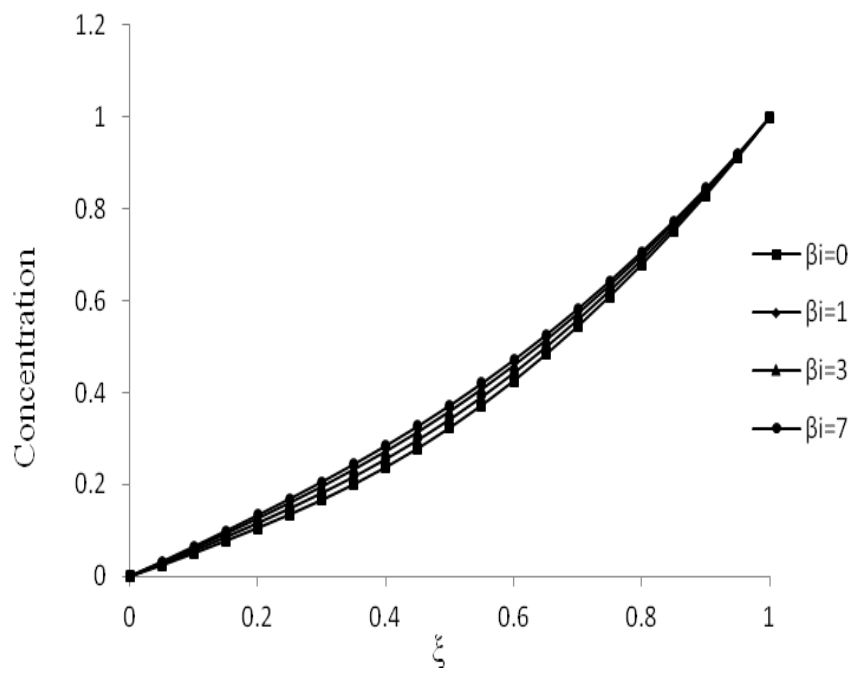

(d)

Fig. 5 Effect of $\beta$ i on (a) Axial Velocity, (b) Radial Velocity, (c) Temperature and (d) Concentration for $\mathrm{Kr}=2, \beta \mathrm{e}=0.2, \mathrm{Gr}=20, \mathrm{Sr}=0.02, \mathrm{Du}=0.2, \mathrm{Gm}=20, \mathrm{Sc}=0.22, \mathrm{Pr}=0.2, \mathrm{Re}=2, \mathrm{D}^{-1}=2, \beta=10, \alpha=0.5, \mathrm{Ha}=10$ 


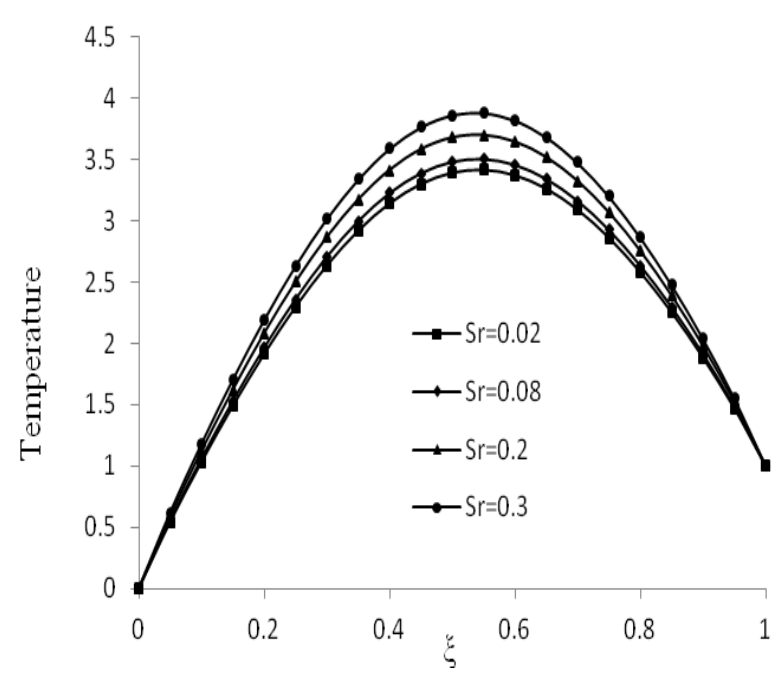

(a)

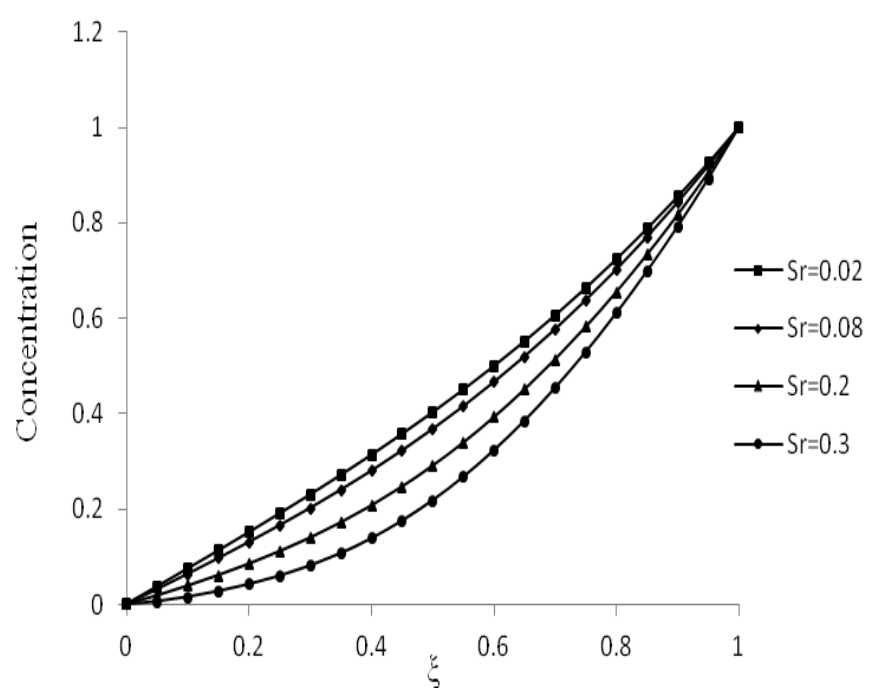

(b)

Fig. 6 Effect of $\mathrm{Sr}$ on (a) Temperature and (b) Concentration for $\mathrm{Kr}=2, \beta \mathrm{e}=0.2, \mathrm{Gr}=10, \beta \mathrm{i}=0.2, \mathrm{Du}=2$, $\mathrm{Gm}=10, \mathrm{Sc}=0.2, \mathrm{Pr}=0.2, \mathrm{Re}=2, \mathrm{D}^{-1}=2, \beta=2, \alpha=0.2, \mathrm{Ha}=2$.

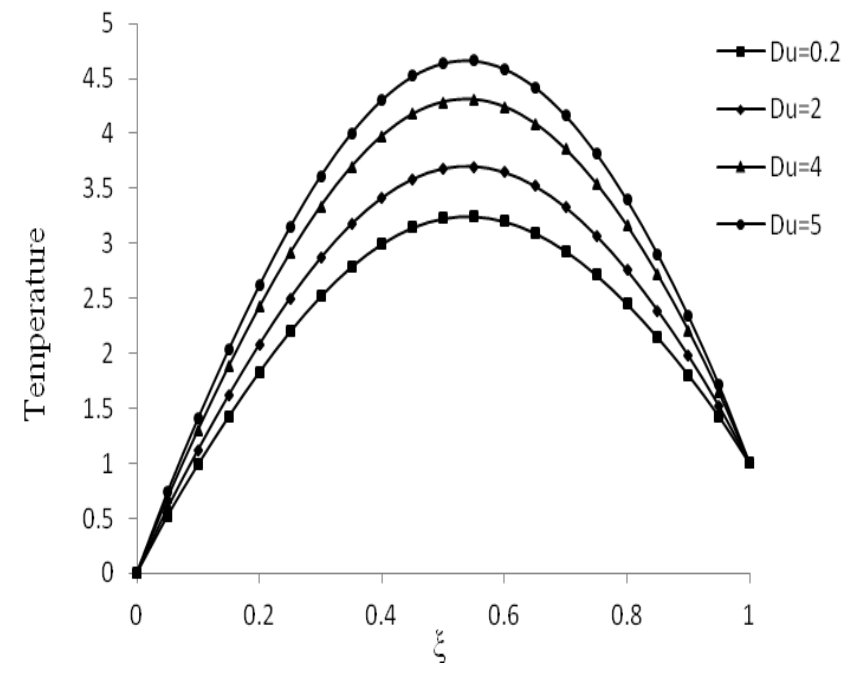

(a)

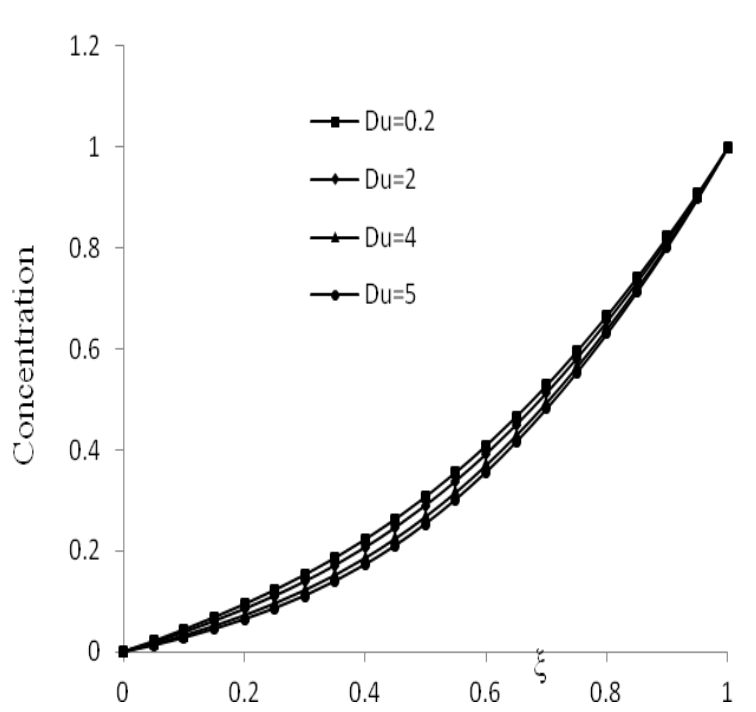

(b)

Fig. 7 Effect of Du on (a) Temperature and (b) Concentrationfor $\mathrm{Kr}=2, \beta \mathrm{e}=0.2, \mathrm{Gr}=10, \beta \mathrm{i}=0.2, \mathrm{Sr}=0.2$, $\mathrm{Gm}=10, \mathrm{Sc}=0.2, \operatorname{Pr}=0.2, \mathrm{Re}=2, \mathrm{D}^{-1}=2, \beta=2, \alpha=0.2, \mathrm{Ha}=2$ 


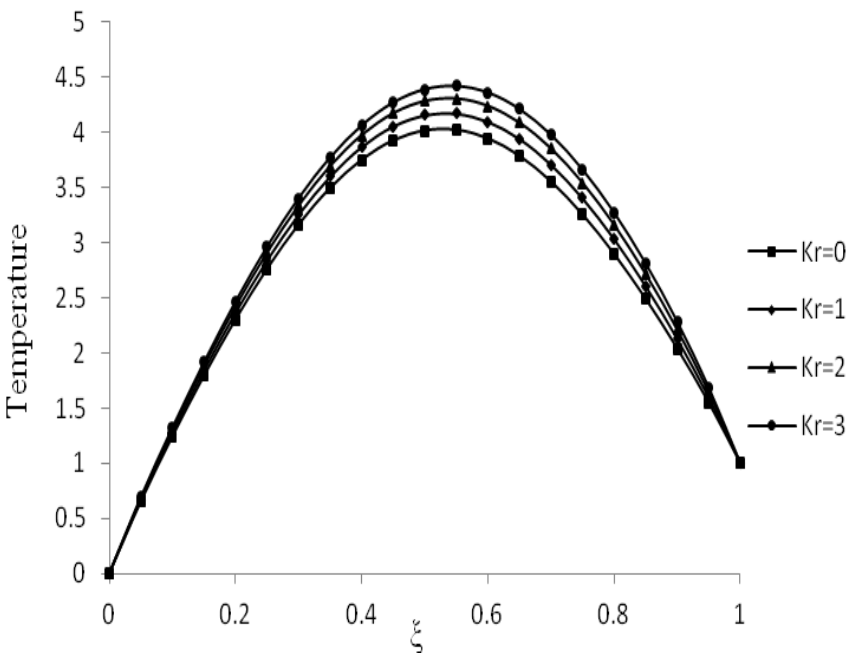

(a)

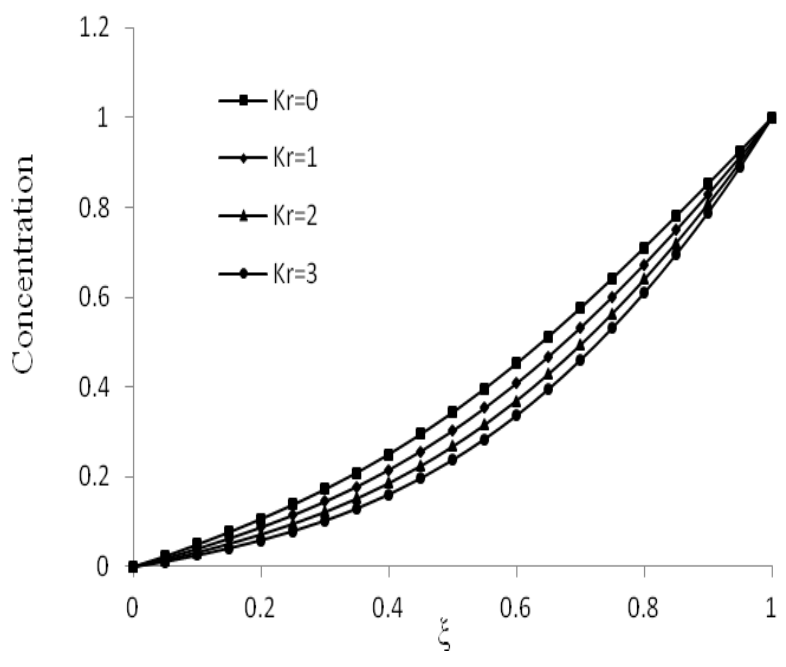

(b)

Fig. 8 Effect of $\mathrm{Kr}$ on (a) Temperature and (b) Concentration for $\mathrm{Du}=4, \beta \mathrm{e}=0.2, \mathrm{Gr}=10, \beta \mathrm{i}=0.2, \mathrm{Sr}=0.2$, $\mathrm{Gm}=10, \mathrm{Sc}=0.2, \mathrm{Pr}=0.2, \mathrm{Re}=2, \mathrm{D}^{-1}=2, \beta=2, \alpha=0.2, \mathrm{Ha}=2$.

\section{CONCLUSIONS}

The influence of the Hall and ion slip on an unsteady free convection heat and mass transfer of an electrically conducting couple stress fluid in a porous medium between expanding or contracting walls with chemical reaction, Soret and Dufour effects is considered. The reduced governing equations are solved numerically by the method of quasilinearization. The results are presented in the form of graphs for different values of fluid and geometric parameters and from these it is concluded that

- The influence of $\mathrm{Sr}$ and $\mathrm{Du}$ on temperature and concentration are similar.

- $\mathrm{Kr}$ reduces the concentration and enhances the temperature of the fluid.

- The velocity components and temperature distribution are exhibiting the same effects for $\mathrm{Gr}$ and $\mathrm{Gm}$.

- The parameters $\mathrm{Sr}$ and $\mathrm{Kr}$ show the opposite effect for temperature and concentration.

\section{NOMENCLATURE}

$\mathrm{t}$

a(t) Distance between parallel plates

$V_{1} \quad$ Suction velocity

Pr Prandtl number, $\mu c / k$

Re Reynolds number, $\rho V_{1} a / \mu$

$\bar{J} \quad$ Current density

$\bar{B} \quad$ Total magnetic field

$\bar{b} \quad$ Induced magnetic field

$B_{0} \quad$ Magnetic flux density

$\bar{E} \quad$ Electric field

Ha Hartmann number, $B_{0} a \sqrt{\sigma / \mu}$
$\mathrm{D}^{-1} \quad$ Inverse Darcy parameter, $a^{2} / k_{1}$

$\mathrm{p} \quad$ Fluid pressure

$\bar{Q} \quad$ Velocity vector

c Specific heat at constant temperature

Ec Eckert number, $\mu V_{1} /\left[\rho a c\left(T_{2}-T_{1}\right)\right]$

$\mathrm{k}$ Thermal conductivity

$k_{1} \quad$ Permeability Parameter

$\mathrm{u} \quad$ Velocity component in $\mathrm{x}$-direction

Velocity component in y-direction

Temperature

$T_{1} \quad$ Temperature of the lower plate

$T_{2} \quad$ Temperature of the upper plate

$T^{*} \quad$ Dimensionless temperature, $\left(T-T_{1}\right) /\left(T_{2}-T_{1}\right)$

D Rate of deformation tensor

C Concentration

$\mathrm{D}_{1} \quad$ Mass diffusivity

$k_{2} \quad$ Chemical reaction rate

$\mathrm{Kr} \quad$ Non dimensional chemical reaction parameter, $k_{2} a^{2} / D_{1}$

Sc $\quad$ Schmidt number, $v / D_{1}$

Gr Thermal Grashof number, $\rho g \beta_{T}\left(T_{2}-T_{1}\right) a^{3} / v^{2}$

$\mathrm{Gm} \quad$ Solutal Grashof number, $g \beta_{C}\left(C_{1}-C_{0}\right) a^{3} / v^{2}$

Sh Sherwood number, $n_{A} /\left[a v\left(C_{1}-C_{0}\right)\right]$

$n_{A} \quad$ Mass transfer rate

Sr Soret number, $D_{1} k_{T} v V_{1} /\left(c T_{m} n_{A}\right)$

$\mathrm{Du} \quad$ Dufour number, $D_{1} k_{T} \dot{n}_{A} \rho c /\left(v^{2} V_{1} c_{s} k\right)$

$T_{m} \quad$ Mean temperature 
$k_{T} \quad$ Thermal-diffusion ratio

$c_{s} \quad$ Concentration susceptibility

$\overline{F_{B}} \quad$ Buoyancy force

$\wedge \wedge \wedge$

$i, j, k$ Unit vectors

\section{Greek Symbols}

$\xi \quad$ Dimensionless y coordinate, $y / a$

$\zeta \quad$ Dimensionless axial variable, $x / a$

$\rho \quad$ Fluid density

$\mu \quad$ Fluid viscosity

$\mu^{\prime} \quad$ Magnetic permeability

$\sigma \quad$ Electric conductivity

$\alpha \quad$ Couple stress parameter, $\sqrt{\eta /\left(\mu a^{2}\right)}$

$\beta_{T} \quad$ Coefficient of thermal expansion

$\beta_{C} \quad$ Coefficient of solutal expansion

$\beta \mathrm{i} \quad$ Ion slip parameter

Be Hall parameter

ae $\quad$ Hall and ion-slip parameter, $1+\beta \mathrm{i} \beta \mathrm{e}$

$\beta \quad$ Wall expansion ratios, $a$ a / $v$

\section{REFERENCES}

Vijay Kumar Stokes, 1966, "Couple Stresses in Fluids," Phys of Fluids, 9, 1709-1715.

http://dx.doi.org/10.1063/1.1761925

Stokes V.K., 1968, "Effects of Couple Stress in Fluid on Hydromagnetic Channel Flow," Phys.of Fluids, 11(5), 1131-1133. http://dx.doi.org/10.1063/1.1692056

Srinivasacharya D., Srinivasacharyulu N., Odelu Ojjela, 2009, "Flow and Heat Transfer of Couple Stress Fluid in a Porous Channel with Expanding and Contracting walls," International Communications in Heat and Mass Transfer, 36 (2), 180-185.

http://dx.doi.org/10.1016/j.icheatmasstransfer.2008.10.005

Najeeb Alam Khan, Amir Mahmood, Asmat Ara, 2013, “Approximate Solution of Couple Stress Fluid with Expanding or Contracting Porous Channel," International Journal for Computer-Aided Engineering and Software, 30(3), 399-408.

http://dx.doi.org/10.1108/02644401311314358

Xin-hui SI, Lian-cun zheng, Xin-xin zhang, Ying chao, 2010, "Perturbation Solution to Unsteady Flow in a Porous Channel with Expanding or Contracting Walls in the Presence of a Transverse Magnetic Field," Applied Mathematics and Mechanics -English Edition, 31(2), 151-158.

http://dx.doi.org/10.1007/s10483-010-0203-z

Xin-Hui Si, Lian-cun Zheng, Xin-Xin Zhang, Ying Chao, 2011, "Homotopy Analysis Solution for the Asymmetric Laminar Flow in a Porous Channel with Expanding or Contracting Walls," Acta Mechanica Sinica, 27 (2), 208-214. http://dx.doi.org/10.1007/s10409-011-0430-3

Uchida S., Aoki H., 1977, "Unsteady Flows in a Semi-infinite Contracting or Expanding Pipe," Journal of Fluid Mechanics, 82, 371381.

http://dx.doi.org/10.1017/S0022112077000718

Srinivas S., Subramanyam Reddy A., Ramamohan T.R., 2012, “A Study on Thermal-diffusion and Diffusion-thermo Effects in a Twodimensional Viscous Flow between Slowly Expanding or Contracting Walls with Weak Permeability," International Journal of Heat and Mass Transfer, 55, 3008-3020.

http://dx.doi.org/10.1016/j.ijheatmasstransfer.2012.01.050

Srinivasacharya D., Kaladhar K., 2011, "Mixed Convection in a Couple Stress Fluid with Soret And Dufour Effects," International Journal of Applied Mathematics and Mechanics, 7(20), 59-71.

Malasetty M.S., Gaikwad S.N., Swamy M., 2006, “An Analytical Study of Linear and Non-linear Double Diffusive Convection with Soret Effect in Couple Stress Liquids," International Journal of Thermal Sciences, 45, 897-907.

http://dx.doi.org/10.1016/j.ijthermalsci.2005.12.005

Dursunkaya Z., Worek W.M., 1992, "Diffusion-thermo and Thermaldiffusion Effects in Transient and Steady Natural Convection from Vertical Surface," International Journal of Heat and Mass Transfer, 35, 2060-2065.

http://dx.doi.org/10.1016/0017-9310(92)90208-A

Mahmoud M.A.A., Megahed A.M., 2013, "Thermal Radiation Effect on Mixed Convection Heat and Mass Transfer of a Non-Newtonian Fluid over a Vertical Surface Embedded in a Porous Medium in the Presence of Thermal-diffusion and Diffusion-thermo Effects, Journal of Applied Mechanics and Technical Physics, 54(1), 90-99. http://dx.doi.org/10.1134/S0021894413010112

Nithyadevi N., Ruey- Jen Yang, 2009, "Double Diffusive Natural Convection in a partially Heated Enclosure with Soret and Dufour Effects," International Journal of Heat and Fluid Flow, 30, 902-910. http://dx.doi.org/10.1016/j.ijheatfluidflow.2009.04.001

Adrian Postelnicu, 2004, "Influence of a Magnetic Field on Heat and Mass Transfer by Natural Convection from Vertical Surfaces in Porous Media considering Soret and Dufour Effects," International Journal of Heat and Mass Transfer, 47, 1467-1472.

http://dx.doi.org/10.1016/j.ijheatmasstransfer.2003.09.017

Dulal Pal, Hiranmoy Mondal, 2011, “MHD Non-Darcian Mixed Convection Heat and Mass Transfer over a Non-linear Stretching Sheet with Soret-Dufour Effects and Chemical Reaction," International Communications in Heat and Mass Transfer, 38, 463-467. http://dx.doi.org/10.1016/j.icheatmasstransfer.2010.12.039

Ganesan P., Suganthi R.K., Loganathan P., 2012, "Soret and Dufour Effects in a Free Convective Doubly Stratified Flow over a Vertical Plate with Chemical Reaction,"Chemical Engineering Communications, 200, 514-531.

http://dx.doi.org/10.1080/00986445.2012.712580

Ching-Yang Cheng, 2010, "Soret and Dufour Effects on Heat and Mass Transfer by Natural Convection from a Vertical Truncated Cone in a Fluid-saturated Porous Medium with Variable Wall Temperature and Concentration," International Communications in Heat and Mass Transfer, 37, 1031-1035.

http://dx.doi.org/10.1016/j.icheatmasstransfer.2010.06.008 
Hayat T., Nawaz M., 2011, "Soret and Dufour Effects on the Mixed Convection Flow of a Second Grade Fluid subject to Hall and Ion-slip Currents," International Journal of Numerical Methods in Fluids, 67, 1073-1099.

http://dx.doi.org/10.1002/fld.2405

Nasser S. Elgazery, 2009, "The Effects of Chemical Reaction, Hall and Ion-slip Currents on MHD Flow with Temperature Dependent Viscosity and Thermal Diffusivity," Communications in Nonlinear Science and Numerical Simulation, 14 (4), 1267-1283.

http://dx.doi.org/10.1016/j.cnsns.2007.12.009

Ali Chamkha, Mansour M.A., Abdelraheem Aly, 2011, "Unsteady MHD Free Convective Heat and Mass Transfer from a Vertical Porous Plate with Hall Current, Thermal Radiation and Chemical Reaction Effects," International Journal of Numerical Methods in Fluids, 65 , 432-447.

http://dx.doi.org/10.1002/fld.2190
Patil P.M., Kulkarni P.S., 2008, "Effects of Chemical Reaction on Free Convective Flow of a Polar Fluid through a Porous Medium in the Presence of Internal Heat Generation," International Journal of Thermal Sciences, 47, 1043-1054.

http://dx.doi.org/10.1016/j.ijthermalsci.2007.07.013

Bhatnagar R., Vayo H.W., Okunbor D., 1994, "Application of Quasilinearization to Viscoelastic Flow through a Porous Annulus," International Journal of Non-Linear Mechanics, 29(1), 13-22.

http://dx.doi.org/10.1016/0020-7462(94)90048-5

Bellman R.E., Kalaba R.E., 1965, Quasilinearization and Boundaryvalue Problems, Elsevier publishing Co. Inc., New York.

Eckert E.R.G., Drake R.M., 1972, Analysis of Heat and Mass Transfer, McGraw-Hill, New York.

Sutton G.W., Sherman A., 1965, Engineering Magnetohydrodynamics, McGrawhill, New York. 